\title{
Self-Compassion in Weight Management: A Systematic Review ${ }^{\dagger}$
}

\author{
Jennifer Brenton-Peters *, Nathan Consedine, Anna Boggis and Anna Serlachius \\ Psychological Medicine, University of Auckland, Auckland 1010, New Zealand; \\ n.consedine@auckland.ac.nz (N.C.); abog579@aucklanduni.ac.nz (A.B.); a.serlachius@auckland.ac.nz (A.S.) \\ * Correspondence: jbre092@aucklanduni.ac.nz; Tel.: +64-022-671-3224 \\ + Presented at the 2019 Annual Meeting of the Nutrition Society of New Zealand, Napier, New Zealand, \\ 28-29 November 2019.
}

Published: 13 December 2019

Every day millions of individuals seek to manage their weight. However, despite weight management efforts, the number of individuals living with overweight and obesity is increasing globally. Excess weight is associated with many chronic diseases that can impact an individual's health and quality of life. Therefore, it is essential that weight management interventions evolve to improve outcomes.

Adding psychological components to weight management interventions is promising. The last 10 years has seen a surge in research on self-compassion (treating oneself with kindness during times of failure or distress) and its links to health outcomes. The objective of this systematic review is to evaluate the evidence assessing whether self-compassion interventions can support weight management outcomes, defined as healthier eating, increased exercise, and improved weight.

Following PRISMA guidelines, databases were searched independently by two reviewers using the keywords: 'self-compassion' or 'selfcompassion' and 'diet', or 'eat' adj 'behaviour', or 'food', or 'exercise' or 'activ*' or 'behav ${ }^{* \prime}$. Studies published in English, peer-reviewed journals, including a self-compassion intervention or self-compassion as a component of an intervention, and reporting weight management outcomes were included.

A total of $\mathrm{N}=20$ studies were identified. Of these studies, ten were pilot or feasibility studies, thirteen were in a population living with overweight and obesity, four were in a clinical population. Participants were predominately female. In most studies, self-compassion was a sub-component of a broader intervention. Improvements were found in all studies that evaluated eating behaviours and physical activity. Six of the thirteen studies reporting weight outcomes demonstrated improvements in weight-loss. Methodological weaknesses and variability in outcome measures limits the ability to make strong conclusions on the evidence of self-compassion in weight management. There is a need for more research on self-compassion specific interventions reporting weight management outcomes and using more diverse populations. 